\title{
Laser-patterned paper-based flow-through filters and lateral flow immunoassays to enable the detection of $\mathrm{C}$-reactive protein
}

P. P. Galanis *, I. N. Katis, P. J. W. He, A. H. Iles, A.J.U. Kumar, R. W. Eason and C. L. Sones

Optoelectronics Research Centre, University of Southampton, Highfield, Southampton, SO17 1BJ, UK

*Corresponding author:

Panagiotis P. Galanis

Email:pg1n15@soton.ac.uk 


\begin{abstract}
We report the use of a laser-based fabrication process in the creation of paper-based flow-through filters that when combined with a traditional lateral flow immunoassay provide an alternative pathway for the detection of a pre-determined analyte over a wide concentration range. The laser-patterned approach was used to create polymeric structures that alter the porosity of the paper to produce porous flow-through filters, with controllable levels of porosity. When located on the top of the front end of a lateral flow immunoassay the flow-through filters were shown to block particles (of known sizes of $200 \mathrm{~nm}, 500 \mathrm{~nm}, 1000$ $\mathrm{nm}$ and $3000 \mathrm{~nm}$ ) that exceed the effective pore size of the filter while allowing smaller particles to flow through onto a lateral flow immunoassay. The analyte detection is based on the use of a size-exclusive filter that retains a complex $(\sim 3 \mu \mathrm{m}$ in size $)$ formed by the binding of the target analyte with two antibodies each of which is tagged with different-sized labels ( $40 \mathrm{~nm}$ Aunanoparticles and $3 \mu \mathrm{m}$ latex beads), and which is larger than the effective pore size of the filter. This method was tested for the detection of C-reactive protein in a broad concentration range from $10 \mathrm{ng} / \mathrm{ml}$ to 100,000 ng/ml with a limit-of-detection found at $13 \mathrm{ng} / \mathrm{ml}$ and unlike other reported methods used for analyte detection, with this technique we are able to counter the Hook effect which is a limiting factor in many lateral flow immunoassays.
\end{abstract}

Keywords: Paper-based device; Pre-polymer; Laser patterning; Porous flow-through filter; CRP detection; Hook effect

\title{
1. Introduction
}

Point-of-care (POC) tests have been widely developed for more than a decade and are intended for near patient diagnostic testing for detection of various conditions and diseases [1]. These diagnostic tests, which can be of vital importance especially in resource-limited settings, are simple to use and can be easily performed by untrained personnel giving results within 30 minutes [2,3]. Any POC diagnostic sensor designed for use in resource-limited settings would need to comply with the criteria set by the World Health Organization (WHO) which address the need to be affordable, sensitive and specific for analyte detection, user friendly, robust and provide the results rapidly to the patients and be equipmentfree and deliverable to those who need it [4]. Paper has been shown to be an attractive platform for the development of POC sensors [5] that would comply with all these WHO-specified requirements. Due to its inherent characteristics (e.g. cost-effectiveness, biocompatibility, wicking of fluids through capillary action etc.), paper has been used extensively, for more than a decade, for the detection of pathogens and it has been established as a material for commercial products in the market $[6,7]$.

Lateral flow immunoassays (LFIAs) are a specific category of POC sensors that have been used for the detection and quantification of analytes within biological fluids such as urine [8], blood [9] and saliva [10]. LFIAs are made up of four constituents: the sample pad, conjugate pad, detection pad and absorbent pad and the principle of operation of an LFIA is based on the interaction (binding) of the target analyte with antibodies. The sample, which contains the analyte, is introduced via the sample pad and after travelling laterally in the porous paper, it first encounters the conjugate pad where immobilized antibodies (detection antibodies) tagged with a coloured label such as Au-nanoparticles bind with the analyte. The sample then flows to the detection pad where antibodies (capture antibodies) specific to the analyte are immobilized at the test line and capture the target analyte thus producing a visual signal. The sample continues flowing past the control line where the detection antibodies are captured (by a different capture antibody specific to the detection antibody) regardless of the presence of the analyte thus producing a visual signal at the control line, showing that the test has performed properly. The excess liquid is finally absorbed in a pad at the end of the LFIA [11].

LFIAs have some unique advantages over other diagnostics, one of the most important being that these devices do not need to be stored in a refrigerator following manufacture, which makes them ideal for use in remote settings and developing countries [12]. Furthermore, the test procedure is relatively simple and produces results which can then be interpreted visually by the patients [13]. Despite their advantages, LFIAs have some drawbacks arising primarily from the detection methods (i.e. chemistries/immunoassays) that are used, and this can limit the range of concentrations in which the analytes can be detected. Secondly, for semi-quantitative analysis, visual evaluation may not be reliable or definitive and therefore readers, cameras or even use of multiple lines as capture sites in the detection pad are reported [14].

For the case where the tested sample contains high analyte concentrations, and of direct relevance to our work here, the detection is limited as a consequence of the Hook effect [15]. This is a result of the increased number of unlabelled analytes which bind to the test line of the LFIA preventing the capture of labelled analytes on the same line. Above a certain concentration, as a result of the Hook effect the colour intensity of the test line decreases with increasing concentration of the analyte, consequently leading to a 
false interpretation of the results. In order to overcome this problem and increase the dynamic range across which the analyte can be detected, methods have been reported that either use an additional line formed of the antigen and positioned between the test line and the control line [16] or use of multiple test lines in the detection pad [17]. These methods can successfully increase the dynamic range in which the analyte can be detected, however they require the use of a conjugate pad and subsequently the incubation and drying of the detection antibodies for several minutes, a step that increases the time for the device fabrication.

Unlike our previously reported method that applied laser-patterned constrictions within the channel of an LFIA [18] for improving the limit-of-detection of a common inflammation marker namely Creactive protein (CRP) [19], in this paper we report a novel filter-based method used for the detection of the same marker over a broad concentration range. The detection of CRP is clinically significant [20], and it is important for it to be measured over a wide concentration range. Although CRP is present in our body, elevated levels are associated with diseases such as chronic inflammation as well as type 2 diabetes mellitus [21]. Common methods used for the detection of CRP [22] include the use of ELISA tests [23] and LFIAs [24]. However, due to the limiting factor of the Hook effect, there is a limitation in the concentration range across which this analyte can be detected. This limitation can be detrimental in the case of CRP detection as it is reported [25] that the levels of this protein are associated with different risk levels for cardiovascular disease ranging widely from $<1000 \mathrm{ng} / \mathrm{ml}$ for low risk to $>3000 \mathrm{ng} / \mathrm{ml}$ for high risk.

To address the aforementioned challenge, it is important to have a device capable of detecting CRP over a broad range spanning from a few $\mathrm{ng} / \mathrm{ml}$ to values greater than $3000 \mathrm{ng} / \mathrm{ml}$. Here we employ a combination of a laser-patterned flow-through filter, and an LFIA to demonstrate such a detection strategy. The flow-through filter was designed to block particles larger than a specific size while allowing smaller particles to flow through the filter and get captured in the test line of a standard LFIA. Both the filters and the LFIAs are made using the principle of light-induced polymerisation of an acrylate-based negative pre-polymer which is locally deposited across an LFIA to create user-defined flow paths. We have previously reported the use of such laser-patterned LFIAs for the detection of a single analyte such as the Leishmaniasis antigen [26] but also in multiplexed detection of analytes [27] as well as bacterial infection testing [28]. In this method, the pre-polymer is deposited on a cellulose paper and after penetration throughout the paper thickness it is polymerised using laser light to form solid polymeric structures of controllable porosity.

The detection strategy is based on the interaction and binding of the target analyte with two antibodies, each tagged with a differently sized label namely $40 \mathrm{~nm}$ Au-nanoparticles and $3 \mu \mathrm{m}$ latex beads. The presence of the analyte in the sample leads to the formation of a complex formed of the analyte and the two antibodies tagged with the small and large particles. This complex is blocked by the flowthrough filter, as its total size is larger than the effective pore size of the filter. In the case of a (i) positive sample with a high analyte concentration, the Au-nanoparticles bind to form the complex which cannot flow through the filter and hence the Au-nanoparticles cannot be captured by the test line on the LFIA, therefore no signal is produced at the test line (similar to a competitive immunoassay). When the analyte is (ii) not present in the sample, i.e. the sample is negative, the complex is not formed and therefore the Au-nanoparticles are free to flow through the filter onto the LFIA and get captured at the test line. So, in the case of a negative sample, a strong coloured red line will appear at the test line (again similar to a competitive immunoassay). For samples of (iii) different analyte concentrations, some of the Aunanoparticles will be captured to form the complex and only the unbound ones will flow through the filter and get captured at the test line. In that case a coloured signal of variable intensity will be produced in the test line, indicating the presence of the analyte in the sample.

Our proposed method is simple and uses the same materials that are used in standard LFIAs. However, unlike the case for standard LFIA operation and other methods reported for improving the dynamic range, we do not make use of the sample pad and the conjugate pad thereby simplifying further the device design and fabrication protocol. Instead, we are using a porous filter which is able to retain particles of a specific size while allowing smaller particles to flow through. We tested this method and were able to measure the presence of CRP over a broad concentration range from $10 \mathrm{ng} / \mathrm{ml}$ to 50,000 ng/ml with a limit-ofdetection found at $13 \mathrm{ng} / \mathrm{ml}$.

Several studies in the literature [16, 29-31] report observing the Hook effect in LFIAs for CRP at concentrations higher than a threshold value of $500 \mathrm{ng} / \mathrm{ml}$. We do not present herein a measurement of 
this threshold for a direct comparison of our flow-through filter-based detection immunoassay with a traditional LFIA, because we cannot use the same detection and capture antibodies for a direct comparison. However, since the three repeats (for each of the different analyte concentration) produce the same answer, our proposed method is able to operate accurately and detect CRP over a broad concentration range from $10 \mathrm{ng} / \mathrm{ml}$ to $50,000 \mathrm{ng} / \mathrm{ml}$ which is beyond the previously mentioned threshold value for the Hook effect.

\section{Experimental Section}

\subsection{Experimental setup and materials}

The porous paper used for the fabrication of the flow-through filters is Whatman ${ }^{\mathrm{TM}}$ grade 1 qualitative filter paper (cellulose) with a thickness of $180 \mu \mathrm{m}$ and pore size of $11 \mu \mathrm{m}$ from GE Healthcare. The part of the sample that flows through the filter ends up in an LFIA and the material of choice for that is a UniSart CN 95 (nitrocellulose) membrane from Sartorius Stedim Biotech GmbH, Germany with thickness of $140-170 \mu \mathrm{m}$ and pore size of $15 \mu \mathrm{m}$. The sample that flows over the test line accumulates in an absorbent pad (Whatman ${ }^{\text {TM }}$ CF4) which is acquired from GE Healthcare and has thickness of $482 \mu \mathrm{m}$. In order to change the porosity of the paper and make the flow-through filters, we used an acrylate-based negative pre-polymer (DeSolite ${ }^{\circledR}$ 3471-3-14) from DSM Desotech, Inc., USA which is deposited using a PICO ${ }^{8} \mathrm{P} \mu 1 \mathrm{se} \mathrm{T}^{\mathrm{TM}}$ dispenser platform from Nordson EFD, UK. The dispensing system, which delivers the pre-polymer, deposits micro-droplets with a volume of $0.5 \mathrm{~nL}$ through an orifice with diameter 100 $\mu \mathrm{m}$ at a frequency of $100 \mathrm{~Hz}$. The light source that is used to cure the pre-polymer is a fibre-coupled continuous wave (c.w.) diode laser (Cobolt MLD, Cobolt AB Sweden) operating at a wavelength of 405 $\mathrm{nm}$ and output power of $60 \mathrm{~mW}$.

In order to create flow-through filters with different levels of porosity, we deposited a solution of the pre-polymer that was diluted with isopropyl alcohol (IPA) (Sigma-Aldrich) in different concentrations. For the characterization of the different porosity filters we used solutions of black dyed polystyrene microspheres (Polysciences, Inc.) of different sizes: $200 \mathrm{~nm}\left(5.68 \times 10^{12}\right.$ particles $/ \mathrm{ml}$, product number $24290-15), 500 \mathrm{~nm}\left(3.64 \times 10^{11}\right.$ particles $/ \mathrm{ml}$, product number $\left.24291-15\right), 1000 \mathrm{~nm}\left(4.55 \times 10^{10} \mathrm{particles} / \mathrm{ml}\right.$, product number $24287-15)$ and $3000 \mathrm{~nm}\left(1.68 \times 10^{9} \mathrm{particles} / \mathrm{ml}\right.$, product number $\left.24292-15\right)$ suspended in deionized water with a concentration of $25 \mathrm{mg} / \mathrm{ml}$. In order to demonstrate the detection mechanism of this filtration method, we used an immunoassay with mouse biotinylated $\operatorname{IgG}(\mathrm{R} \& \mathrm{D}$ systems with stock concentration of $500 \mu \mathrm{g} / \mathrm{ml}$, product number IC002B) as the analyte, anti-mouse antibody (R\&D systems with stock concentration of $500 \mu \mathrm{g} / \mathrm{ml}$, product number G-202-C) labelled with $40 \mathrm{~nm}$ Au-nanoparticles (acquired from Abcam with an optical density 20, product number ab269932) as the detection antibody and $3 \mu \mathrm{m}$ latex beads (white dyed) labelled with streptavidin as the filtration antibody (acquired from Spherotech Inc. with stock concentration of $500 \mu \mathrm{g} / \mathrm{ml}$, product number SVP-30-5). The mouse IgG (R\&D systems with stock concentration of $500 \mu \mathrm{g} / \mathrm{ml}$, product number AF007) was dispensed and immobilized as a capture antibody for the test line on the LFIA. We tested the performance of the flowthrough filters with an immunoassay for the detection of CRP. For this we used (as the analyte) human CRP (Sigma-Aldrich with stock concentration of $1 \mathrm{mg} / \mathrm{ml}$, product number C1617), mouse anti-human CRP (acquired from R\&D systems with stock concentration of $500 \mu \mathrm{g} / \mathrm{ml}$, product number MAB17071) labelled with $40 \mathrm{~nm}$ Au-nanoparticles (as the detection antibody) and mouse anti-human CRP biotinylated IgG antibody (R\&D systems with stock concentration of $50 \mu \mathrm{g} / \mathrm{ml}$, product number BAM17072) tagged with $3 \mu \mathrm{m}$ latex beads (white dyed) labelled with streptavidin as the filtration antibody. Goat anti-mouse $\mathrm{IgG}$ (R\&D systems with stock concentration of $500 \mu \mathrm{g} / \mathrm{ml}$, product number G-202-C) was used at the test line to capture the detection antibody. At the end of the testing process the LFIAs were washed with 10 $\mu \mathrm{L}$ of phosphate-buffered saline to ensure that all the sample had reached the position of the test line. The antibodies were immobilized on the test line of the LFIA using a XYZ3210 platform with a Biojet HR solenoid dispenser from Biodot.

\subsection{Methods and procedure}

The method for the fabrication of solid polymeric walls in both cellulose paper-based flow-through filter and nitrocellulose membrane of the LFIA, involves the use of an acrylate-based negative prepolymer, which is locally deposited on top of these porous substrates before the exposure to a laser light source. The schematic in figure 1 illustrates the setup used to pattern the porous substrates and create the paper-based devices i.e. the flow-through filter and the LFIA. First, the pre-polymer is locally deposited 
by a dispenser on top of a porous substrate at locations pre-defined by a computer design to demarcate the boundaries of the device. The pre-polymer is then allowed to soak for a sufficient time (typically between 1 and 3 minutes depending on the substrate of choice) throughout the entire thickness of the substrate before exposure to the laser light source to induce photo-polymerisation. The laser traces exactly the same pre-polymer pattern previously deposited and the photo-polymerization creates a solid polymeric structure able to contain the flow of liquids. The precise time delay, defined as the difference in time between the pre-polymer deposition and the laser scanning (exposure) depends on the properties of the porous substrate on which the pre-polymer is deposited.

\section{Figure 1}

Figure 1 Schematic representation of the local pre-polymer deposition setup. In this image, the prepolymer is locally deposited onto a porous substrate (nitrocellulose membrane) followed by the subsequent exposure to a fiber-coupled c.w. laser source to create solid polymeric structures/walls that define the fluid-flow path of a lateral flow device.

Figure 2 illustrates (A) a schematic showing the different stages of the fabrication of a porous flowthrough filter as well as (B) a three-dimensional schematic of the same filter positioned on the top of the front end of an LFIA. For the fabrication of a square-shaped frame that forms the impregnable polymeric wall of our filter, the pre-polymer is first deposited as a square-frame (stage 1) on the cellulose paper with a speed of $20 \mathrm{~mm} / \mathrm{s}$. After a time delay of $180 \mathrm{~s}$, which was sufficient for the pre-polymer to penetrate throughout the entire thickness of the paper, the pre-polymer along with the square frame was exposed to the laser irradiation with the same scanning speed of $20 \mathrm{~mm} / \mathrm{s}$ (laser spot size of $2 \mathrm{~mm}$ with a laser fluence of $0.15 \mathrm{~J} / \mathrm{cm}^{2}$ ). During this time delay, the pre-polymer spreads laterally to create an impregnable square frame with a width of $\sim 1 \mathrm{~mm}$ and this therefore defines the minimum width of the solid polymer wall created in the cellulose paper. It is important to note here that the laser follows the same pattern as the pre-polymer that was previously deposited and that is because the set values of the laser spot size and the laser fluence are sufficient to fully polymerize the pre-polymer. Any increase in the laser spot size or change in the laser scanning pattern will result in a decrease of the laser fluence and consequently it would take longer to polymerize any given area or structure which would be detrimental to the overall manufacturing process as it would introduce a potential uncertainty to the post-deposition polymerization step.

\section{Figure 2}

Figure 2 (A) Schematic showing the various steps involved in the fabrication and use of the porous filter with an LFIA: the creation of a solid polymeric structure on a porous substrate (stage 1), pipetting of 10 $\mu \mathrm{L}$ of the diluted pre-polymer inside the solid polymeric structure (stage 2), laser light exposure of the diluted pre-polymer to create the flow-through filter (stage 3) and finally the position of this filter on the top of the front end of an LFIA (stage 4). (B) Three-dimensional schematic showing both the porous flowthrough filter and the LFIA.

In order to make the porous flow-through filters with controllable levels of porosity, we used the same pre-polymer but this time diluted in IPA in different concentrations. The porosity of the flowthrough filters is defined by the volume concentration of the deposited pre-polymer solution in IPA and by changing the $\mathrm{v} / \mathrm{v} \%$ concentration, we were able to create filters with different porosities. The fabrication process involves pipetting $10 \mu \mathrm{L}$ of the diluted pre-polymer inside the solid polymeric structure (stage 2) and exposing the filter after a time delay of $60 \mathrm{~s}$ to laser light (stage 3) with the same fluence as before (with a laser spot size of $7 \mathrm{~mm}$ ) to induce photo-polymerization and create the porous flow-through filter. The time delay of $60 \mathrm{~s}$ is sufficient for the diluted pre-polymer to extend throughout its whole thickness. As can be seen, the time delay of $60 \mathrm{~s}$ is smaller than the time delay of $180 \mathrm{~s}$ that we used to make the solid square polymeric structure on the same substrate. This difference stems from the fact that the pre-polymer that we used to make the porous filter is diluted in IPA and as a result it has lower viscosity and penetrates faster throughout the paper. Pipetting the pre-polymer in different concentrations on cellulose paper and exposing to laser light will result in the creation of porous filters with correspondingly different levels of permeability for subsequent liquid sample flow. Flow-through filters made with higher pre-polymer concentrations will be less porous compared with flow-through 
filters made with lower pre-polymer concentrations as the concentration of the pre-polymer in each case dictates the resultant porosity of the filters.

Finally, using the same acrylate-based pre-polymer we patterned the LFIA on the nitrocellulose membrane. The patterning conditions remained unaltered during the device fabrication work (prepolymer deposition speed $20 \mathrm{~mm} / \mathrm{s}$ and laser scanning speed $20 \mathrm{~mm} / \mathrm{s}$ ). However, due to the different porosity, thickness and internal structure of the nitrocellulose membrane, which has a porous sponge-like internal structure compared with the fibrous internal structure of the cellulose paper the time delay is different and after extended studies [32] was set to be $30 \mathrm{~s}$ for the nitrocellulose membrane.

After the fabrication, the flow-through filter is cut around its solid boundary walls and then positioned on the top of the front end of an LFIA (stage 4) before a sample is deposited onto the flow-through filter. The key point to note is that the porosity of the flow-through filter is controllable and can be designed to retain particles larger than a user-defined size and allow smaller particles to flow through and into the LFIA.

\section{Results and discussion}

\subsection{Characteristic study of the filtration properties of the porous flow-through filters}

Images of the fabricated devices are depicted in Figure 3. The porous flow-through filters are designed to have an area of $\sim 25 \mathrm{~mm}^{2}$ whereas the front end of the LFIA was designed to have a square shape as well but with an area of $49 \mathrm{~mm}^{2}$ and a channel with length of $20 \mathrm{~mm}$ and width of $3 \mathrm{~mm}$. As can be clearly seen, the square front end of the LFIA was deliberately designed to have an area bigger than that of the flow-through filter so that the porous filter will totally fit inside enabling intimate contact between the two substrates.

\section{Figure 3}

Figure 3 Images of (A) a flow-through porous filter and (B) an LFIA with their respective dimensions. A porous filter is able to retain particles larger than a specific size while allowing smaller particles to flow through and into the LFIA.

To study the filtration properties of the porous flow-through filters, we fabricated identical LFIAs and filters with different porosities. Figure 4 shows an example of porous flow-through filters tested with $10 \mu \mathrm{L}$ of black dyed polystyrene microspheres with a size of $1000 \mathrm{~nm}$. Each of the filters has a different level of porosity starting from a very porous (5.8\% of pre-polymer in IPA) to a less porous filter (11.1\% of pre-polymer in IPA) and each one was replicated and tested three times. As a reference, we provide the results of a blank filter (no pre-polymer added) which proved incapable of retaining the $1000 \mathrm{~nm}$ polystyrene microspheres and consequently allowed them to flow through the filter and into the LFIA. All the flow-through filters were positioned on the top of the front end of the LFIA during the testing process as shown in figure $2 \mathrm{~B}$ and only removed after the end of the experiment to capture photos as presented in figure 4A.

\section{Figure 4}

Figure 4 Images showing flow-through filters tested with $10 \mu \mathrm{L}$ of black dyed polystyrene microspheres of size $1000 \mathrm{~nm}$. The LFIAs are all identical, but the flow-through filters are made with different prepolymer concentrations ranging from $0 \%$ to $11.1 \%$ of pre-polymer in IPA. Images show (A) the flowthrough filters and the LFIAs immediately after the testing process and (B) the same LFIAs after the evaporation of the deionized water.

In figure $4 \mathrm{~A}$, we observe that a low porosity filter made with pre-polymer concentration at $11.1 \%$ is able to fully retain the polystyrene microspheres. However, using a higher porosity filter made with prepolymer concentration between $9.1 \%$ and $7.7 \%$, we are able to retain the microspheres in the porous filter 
and at the same time allow the deionized water, in which the microspheres are dispersed, to flow through the filter and end up in the LFIA. An even higher porosity filter made with pre-polymer concentration below $7.7 \%$, is porous to $1000 \mathrm{~nm}$ polystyrene microspheres thus enabling the flow of this particle size through the filter. As we can see in figure $4 \mathrm{~A}$, we have the formation of a dark region on top of the filters after the completion of the filtration process. This is a result of the porous filters which delay the flow of the samples and due to interaction forces between the polystyrene particles, the microspheres aggregate to form a complex bigger than the porosity of the filter and are therefore unable to flow through. Since for the filters with pre-polymer concentration at $9.1 \%$ and below the deionized water flows through (figure $4 \mathrm{~A}$ ), it is difficult to visualize whether the polystyrene microspheres are retained by the filter or flowed into the LFIA. For that reason, photos of the same LFIAs were taken after the evaporation of the deionized water (figure 4B) in order to visually assess the ability of the porous flow-through filters to block particles larger than a specific size. Apart from testing with polystyrene microspheres with $1000 \mathrm{~nm}$ size, we also tested the flow-through filters with polystyrene microspheres of different sizes namely $200 \mathrm{~nm}, 500 \mathrm{~nm}$ and $3000 \mathrm{~nm}$, all dispersed in deionized water. For each of the different particle sizes and filter porosities, we fabricated and tested three identical devices and the results after testing each one of the devices with $10 \mu \mathrm{L}$ of the solution are all depicted in table 1 . Based on the ability of the porous flow-through filters to block particles of different sizes, we defined a transitional range for the concentration (indicated with a red box in table 1) for which the porous filters transition from being completely impermeable to being permeable to polystyrene particles of a specific size. Looking at this transitional range we observe that there is an increasing trend in terms of the $\mathrm{v} / \mathrm{v} \%$ concentration of the pre-polymer and the ability of the filters to block particles decreasing in size.

\section{Table 1}

Table 1 A range of flow-through filters with different porosities for which polystyrene microspheres of different sizes will pass through. For each polystyrene particle size, we have defined a transitional range of pre-polymer concentration (marked in red) in which the filters transition from being completely impermeable to being porous.

In figure 5, we have plotted the particle filtration efficiency of flow-through filters with different porosities after testing with polystyrene microspheres of varying sizes. The particle filtration efficiency is a measure of the performance of a porous flow-through filter when filtering out particles of a specific size. In order to calculate the values for the particle filtration efficiency, we performed colour analysis using Adobe Photoshop. Specifically, we used the RGB channel in the histogram function and measured the mean intensity value of the pixels in both the filters (figure 4A) and the LFIAs (figure 4B).

\section{Figure 5}

Figure 5 Particle filtration efficiency of flow-through filters made with different pre-polymer concentrations and tested with polystyrene microspheres of varying sizes.

We then used equation 1 to calculate the particle filtration efficiency (\%) for the different filter porosities. A value of 255 corresponds to the pixel intensity for white, which is the background of both the flow-through filter and the LFIA before testing. We subtracted the mean colour intensities (for the total of three devices) of both the flow-through filters and the lateral flow-devices from this value in order to obtain the actual signal intensities.

$$
(1-[(255-\text { colour intensity on device }) /(255 \text { - colour intensity on filter })]) \times 100
$$

Using this methodology, we can use such flow-through filters and the principle of size exclusive filtration as an immunoassay for the detection of an analyte, and this is described in the subsequent sections. 


\subsection{Flow-through filtration for analyte detection}

These porous flow-through filters were then used with standard LFIAs for the detection of an analyte using an immunoassay which is based on particle size selection. For this we made the flow-through filter and the lateral flow devices with materials commonly used in LFIAs such as cellulose paper (sample pad), nitrocellulose membrane (detection pad) and absorption pad. Unlike a standard LFIA configuration, in this case we do not make use of the sample or conjugate pads. Instead, we use a porous flow-through filter, which is located on the top of the front end of an LFIA and by altering its porosity, we can design it to retain particles above a certain size. The analyte detection is based on the filtration of different-sized Au-nanoparticles $(40 \mathrm{~nm})$ and latex beads $(3 \mu \mathrm{m})$ that both bind to the same target analyte via antibodies. In this immunoassay, the filters were designed to have a defined pore size bigger than the size of the Au-nanoparticles and thus the particles are able to flow through and reach the test line, whereas the latex beads which have size bigger than the effective pore size of the filters are blocked or retained in the filters. Both Au and latex particles are captured by the analyte and therefore if the analyte is present in the sample (positive sample) it will bind to both and form a complex ( $\sim \mu \mathrm{m}$ in size), which is bigger than the pore size of the filter and as a result is unable to flow through and reach the test line. Only the unbound detection antibodies left in the sample will be able to flow through the filter and get captured at the test line of the LFIA producing a red-coloured line indicating the presence of the analyte in the sample. Similar to the principle of a competitive immunoassay, a test line displaying a clear signal means that the analyte is not present in the sample. For our filter-based immunoassay, an increase in the concentration of the analyte will result in a decrease of the number of unbound detection antibodies consequently captured at the test line, and this therefore will result in a decreased signal at the test line. In the schematic of figure 6 we describe the principle of operation of the filterbased immunoassay in the cases of no analyte, low and high analyte concentrations.

\section{Figure 6}

Figure 6 Schematic representation of the method used for the detection of an analyte. For the case where the analyte in not present in the sample all the detection antibodies are captured at the test line producing a strong signal. However, as we increase the concentration of the analyte, the number of the unbound detection antibodies left in the sample decreases and this will result in a decrease of the signal at the test line.

In our first demonstration of this filtration-based immunoassay, we tested a sample that contained a 1:1:1 ratio of anti-mouse antibody labelled with $40 \mathrm{~nm} \mathrm{Au-nanoparticles} \mathrm{(diluted} \mathrm{in} \mathrm{phosphate-buffered}$ saline to have a concentration at $100 \mu \mathrm{g} / \mathrm{ml}$ used as a detection antibody), $3 \mu \mathrm{m}$ latex beads (labelled with streptavidin (stock concentration) used as a filtration antibody) and finally a mouse biotinylated IgG antibody as the target analyte (tested in different concentration from $0 \mu \mathrm{g} / \mathrm{ml}-500 \mu \mathrm{g} / \mathrm{ml}$ ). In the channel of the LFIAs, we dispensed and immobilized mouse IgG antibody (stock concentration) as the test line (figure 6,7 ). There is no a-priori rationale for the choice of the use of the detection antibody, the filtration antibody, and the analyte on the tested sample in an equal ratio. However, we are well aware that any alteration of this ratio would change the interaction and binding of the target analyte with the detection and the filtration antibodies and therefore result in a change in the number of the complexes that are being formed. In that case, the number of the unbound detection antibodies left in the sample would change, and this would not only affect the colour intensity of the test line of the LFIA but also the concentration range in which the analyte can be detected.

For this immunoassay we fabricated and tested with a $20 \mu \mathrm{L}$ sample a flow-through filter made with 7.7\% pre-polymer concentration (figure 7). For this case we replicated and tested the devices three times. From the results in figure 7 we observe that on using a filter with $7.7 \%$ pre-polymer concentration that is capable of completely retaining particles with a size of $3 \mu \mathrm{m}$, we were able to detect the target analyte in a concentration ranging between $0.5 \mu \mathrm{g} / \mathrm{ml}$ and $500 \mu \mathrm{g} / \mathrm{ml}$. In this case, we observe that an increase in the analyte concentration results in a decrease of the signal at the test line and figure 8 plots the colour intensity of the test line versus the analyte concentration. Each data point is the mean value from the total of three devices. As previously stated, the presence of the analyte in the sample will lead to the formation of the following complex: detection antibody, filtration antibody and mouse biotinylated IgG antibody and the increase in the concentration of the analyte will lead to an increase in the number of such complexes that are being formed. Testing the sample with a porous filter $(7.7 \%$ pre-polymer concentration) the complex thus formed is completely retained in the filter and only the unbound detection antibodies flow through and get captured at the test line indicating the presence of the analyte on the tested sample. The results presented in figure 4 , figure 7 and figure 9 suggest that there is no associated non- 
specific binding of either the detection antibody or the filtration antibody with the porous filter. If that were the case, then the latex beads would not be able to flow through the porous filters unlike the case presented in figure 4. Additionally, in the cases illustrated in figure 7 and figure 9, some of the unbound detection antibodies would non-specifically bind to the porous filter and thus would not be able to flow through it and get captured by the capture antibodies at the test line of the LFIA. As a result, we would not be able to distinguish between a low and a high analyte concentration which has enabled us to detect the analyte over a broad concentration range. Lastly, we cannot be sure if the analytes can non-specifically bind to the polymeric structure of the flow-through filters, however this will have no effect to the immunoassay as the appearance of the test line depends only on the number of the unbound detection antibodies left in the sample.

\section{Figure 7}

Figure 7 Flow-through filter with pre-polymer concentration of $7.7 \%$ tested with $20 \mu \mathrm{L}$ of a sample that contains anti-mouse antibody labelled with $40 \mathrm{~nm}$ Au-nanoparticles, $3 \mu \mathrm{m}$ latex beads labelled with streptavidin and mouse biotinylated $\mathrm{IgG}$ antibody (analyte) at concentrations between $0 \mu \mathrm{g} / \mathrm{ml}$ and 500 $\mu \mathrm{g} / \mathrm{ml}$.

\section{Figure 8}

Figure 8 Colour intensity on the test line of LFIAs for different mouse biotinylated IgG (analyte) concentrations in the range between $0.5 \mu \mathrm{g} / \mathrm{ml}$ and $500 \mu \mathrm{g} / \mathrm{ml}$. The analyte was detected using a flowthrough filter with $7.7 \%$ pre-polymer concentration.

\subsection{Filter-based immunoassay for CRP detection}

The performance of the flow-through filters was tested using an immunoassay for the detection of the common inflammation marker C-reactive protein (CRP). Similar to the previous immunoassay, we used a sample which contains the following constituents mixed in a 1:1:1 ratio: human CRP (analyte) in concentrations ranging from $10 \mathrm{ng} / \mathrm{ml}$ to $100,000 \mathrm{ng} / \mathrm{ml}$, mouse anti-human CRP antibody labelled with $40 \mathrm{~nm}$ Au-nanoparticles (stock concentration) used as a detection antibody and mouse anti-human CRP biotinylated IgG antibody tagged with $3 \mu \mathrm{m}$ latex beads labelled with streptavidin (stock concentration) used as a filtration antibody. The principle of operation of this immunoassay is the same as the one previously described. If the analyte, CRP, is present in the sample, it binds with both the detection antibody and the filtration antibody forming a complex with size bigger than the effective pore size of the filter. Hence this complex is unable to flow through and only the unbound detection antibodies left in the sample are able to flow through the filter and enter the LFIA where they are captured at the test-line (by the goat anti-mouse IgG antibodies) producing a red coloured line. The remaining part of the sample, which is not captured flows over the test line and accumulates in an absorbent pad located at the end of the LFIA (figure 9). The sample, which contains the analyte in different concentrations, was tested on a filter with $7.7 \%$ pre-polymer concentration. Our choice to use a flow-through filter with the given porosity stems from the fact that such a filter is capable of completely retaining particles with size bigger than 3 $\mu \mathrm{m}$ (see table 1), and therefore is able to detect an analyte in a broad dynamic concentration range (figure 7).

In figure 9, we present the results when $20 \mu \mathrm{L}$ of sample containing the CRP analyte in different concentrations $(0-100,000 \mathrm{ng} / \mathrm{ml})$ was tested on a porous flow-through filter and figure 10 depicts the respective colour intensity on the test line where the intensity of the control device $(0 \mathrm{ng} / \mathrm{ml})$ corresponds to a mean value (from the total of three devices) at 46.18 a.u. In this graph, each data point represents the mean value of three devices that were tested. As can be clearly seen, the colour of both the filter and the test line change according to the concentration of the analyte. Similar to our previous experiment, an increase in the CRP concentration will lead to an increase in the number of the complexes (CRP-detection antibody-filtration antibody) that are being formed thus leaving a smaller amount of unbound detection antibodies in the sample which flow through the filter and get captured on the test line of the LFIA. As a result, an increase in the concentration of the analyte will result in a decrease in the signal on the test line 
and using this filter-based method we are able to detect the CRP with a limit-of-detection found at 13 $\mathrm{ng} / \mathrm{ml}$.

\section{Figure 9}

Figure 9 Images of flow-through filters made with $7.7 \%$ pre-polymer concentration and tested with 20 $\mu \mathrm{L}$ of a sample containing varying CRP concentrations from $0 \mathrm{ng} / \mathrm{ml}$ to $100,000 \mathrm{ng} / \mathrm{ml}$. Particles that flow through the filter end up in an LFIA where anti-mouse IgG antibodies have been dispensed and used as a test line.

\section{Figure 10}

Figure 10 Colour intensity on the test line of the LFIA for various CRP concentrations in the range between $10 \mathrm{ng} / \mathrm{ml}$ and 100,000 $\mathrm{ng} / \mathrm{ml}$.

In order to measure the colour intensity in the test line of the LFIAs (figure 7, figure 9) we used again the RGB channel in the histogram function of Adobe Photoshop and we measured the mean intensity value (from the total of three devices) of the pixels of the test line. This value was subtracted from the value of 255 which is the intensity value (from the total of three devices) of the pixels corresponding to the background of the LFIAs (white) in order to obtain the actual signal intensities. For the calculation of the limit-of-detection we used equation 2 [33]:

$$
\mathrm{LOD}=3.3 \times(\sigma / \mathrm{s})
$$

where LOD refers to the limit-of-detection, $\sigma$ refers to the standard deviation of the regression line and $\mathrm{s}$ refers to the slope of the calibration curve. The values of $\sigma$ and $\mathrm{s}$ were both measured from the graph depicted in figure 10 and calculated at 4.3 and 1.1 respectively.

\section{Conclusion}

We have reported a laser-based technique for the fabrication of porous flow-through filters, on a paper substrate, with different levels of porosity that are able to retain particles of a specific size while allowing smaller particles to flow through. The filtration properties and the particle retention efficiency of the flowthrough filters were studied by testing polystyrene microspheres of different sizes and we have successfully found the parameters to make filters able to completely block or allow the flow of the different particle sizes. This filtration technique was later used as an analyte detection mechanism based on the binding of the target analyte with two antibodies, each one tagged with a differently-sized label namely $40 \mathrm{~nm}$ Au-nanoparticles and $3 \mu \mathrm{m}$ latex beads. The antibody-antigen interaction leads to the formation of a complex, which is unable to flow through the filter of a certain porosity when the analyte is present in the sample. Only the unbound detection antibodies with a size smaller than the porosity of the filter can flow through and get captured at the test line of the LFIA indicating the presence or absence of the analyte in the sample. The flow-through filtration method provides an alternative pathway compared to standard LFIAs for the detection of analytes. Unlike other methods reported for the fabrication of standard LFIAs, our proposed technique uses a porous flow-through filter located on the top of the front end of an LFIA which is able to remove particles of a specific size. Using this method, we are able to detect analytes in a concentration range which is beyond the Hook effect threshold, thus avoiding this limiting factor that many analyte detection methods encounter. This method was tested for the detection of a CRP marker covering a broad concentration range between $10 \mathrm{ng} / \mathrm{ml}$ and 100,000 ng/ml, with a limit-of-detection of $13 \mathrm{ng} / \mathrm{ml}$. We believe that this size-exclusive filtration method can be further studied for different applications. Apart from the detection of analytes the porous flow-through filters can also be used as a tool to remove particles which otherwise can be captured in the test line producing false negative results. This application has the potential to improve the performance as well as the limit-ofdetection of the LFIAs and this will be part of our future work. 


\section{Conflicts of interest}

There are no conflicts of interest to declare.

\section{Acknowledgements}

The authors acknowledge the funding received via the Engineering and Physical Sciences Research Council (EPSRC) via Grant Nos. EP/P025757/1, EP/N004388/1, EP/M027260/1 and EP/S003398/1. The underpinning RDM data for this paper can be found at 10.5258/SOTON/D1758

\section{References}

[1] G.J. Fermann, J. Suyama, Point of care testing in the Emergency Department, Journal of Emergency Medicine. 22 (2002) 393-404. https://doi.org/10.1016/s0736-4679(02)00429-8.

[2] P. von Lode, Point-of-care immunotesting: Approaching the analytical performance of central laboratory methods, Clinical Biochemistry. 38 (2005) 591-606. https://doi.org/10.1016/j.clinbiochem.2005.03.008.

[3] T.R. Kozel, A.R. Burnham-Marusich, Point-of-Care Testing for Infectious Diseases: Past, Present, and Future, Journal of Clinical Microbiology. 55 (2017) 2313-2320. https://doi.org/10.1128/jcm.00476-17.

[4] S. Smith, J.G. Korvink, D. Mager, K. Land, The potential of paper-based diagnostics to meet the ASSURED criteria, Rsc Advances. 8 (2018) 34012-34034. https://doi.org/10.1039/c8ra06132g.

[5] A. Abdollahi-Aghdam, M.R. Majidi, Y. Omidi, Microfluidic paper-based analytical devices ( $\mu$ PADs) for fast and ultrasensitive sensing of biomarkers and monitoring of diseases, Biolmpacts: BI. 8 (2018) 237.

[6] K. Yamada, H. Shibata, K. Suzuki, D. Citterio, Toward practical application of paper-based microfluidics for medical diagnostics: state-of-the-art and challenges, Lab on a Chip. 17 (2017) 1206-1249. https://doi.org/10.1039/c6lc01577h.

[7] T. Akyazi, L. Basabe-Desmonts, F. Benito-Lopez, Review on microfluidic paper-based analytical devices towards commercialisation, Analytica Chimica Acta. 1001 (2018) 1-17. https://doi.org/10.1016/j.aca.2017.11.010.

[8] M.D. Moreno, A. Cebolla, A. Munoz-Suano, C. Carrillo-Carrion, I. Comino, A. Pizarro, F. Leon, A. Rodriguez-Herrera, C. Sousa, Detection of gluten immunogenic peptides in the urine of patients with coeliac disease reveals transgressions in the gluten-free diet and incomplete mucosal healing, Gut. 66 (2017) 250-257. https://doi.org/10.1136/gutjnl-2015-310148.

[9] E.C. Schramm, N.R. Staten, Z. Zhang, S.S. Bruce, C. Kellner, J.P. Atkinson, V.C. Kyttaris, G.C. Tsokos, M. Petri, E.S. Connolly, P.K. Olson, A quantitative lateral flow assay to detect complement activation in blood, ANALYTICAL BIOCHEMISTRY. 477 (2015) 78-85. https://doi.org/10.1016/j.ab.2015.01.024.

[10] A. Carrio, C. Sampedro, J. Luis Sanchez-Lopez, M. Pimienta, P. Campoy, Automated Low-Cost Smartphone-Based Lateral Flow Saliva Test Reader for Drugs-of-Abuse Detection, SENSORS. 15 (2015) 29569-29593. https://doi.org/10.3390/s151129569. 
[11] E.B. Bahadir, M.K. Sezginturk, Lateral flow assays: Principles, designs and labels, Trac-Trends in Analytical Chemistry. 82 (2016) 286-306. https://doi.org/10.1016/j.trac.2016.06.006.

[12] G.A. Posthuma-Trumpie, J. Korf, A. van Amerongen, Lateral flow (immuno) assay: its strengths, weaknesses, opportunities and threats. A literature survey, ANALYTICAL AND BIOANALYTICAL CHEMISTRY. 393 (2009) 569-582. https://doi.org/10.1007/s00216-008-22872.

[13] K.M. Koczula, A. Gallotta, Lateral flow assays, in: P. Estrela (Ed.), BIOSENSOR TECHNOLOGIES FOR DETECTION OF BIOMOLECULES, PORTLAND PRESS LTD, 5TH FL, 90 HIGH HOLBORN, LONDON, WC1V 6L, ENGLAND, 2016: pp. 111-120. https://doi.org/10.1042/EBC20150012.

[14] J.H. Cho, S.H. Paek, Semiquantitative, bar code version of immunochromatographic assay system for human serum albumin as model analyte, Biotechnology and Bioengineering. 75 (2001) 725-732. https://doi.org/10.1002/bit.10094.

[15] G.M.S. Ross, D. Filippini, M.W.F. Nielen, G.I.J. Salentijn, Unraveling the Hook Effect: A Comprehensive Study of High Antigen Concentration Effects in Sandwich Lateral Flow Immunoassays, Analytical Chemistry. 92 (2020) 15587-15595.

[16] Y.K. Oh, H.A. Joung, H.S. Han, H.J. Suk, M.G. Kim, A three-line lateral flow assay strip for the measurement of $\mathrm{C}$-reactive protein covering a broad physiological concentration range in human sera, Biosensors and Bioelectronics. 61 (2014) 285-289. https://doi.org/10.1016/j.bios.2014.04.032.

[17] J. Hu, J.R. Choi, S. Wang, Y. Gong, S. Feng, B. Pingguan-Murphy, T.J. Lu, F. Xu, Multiple test zones for improved detection performance in lateral flow assays, Sensors and Actuators B: Chemical. 243 (2017) 484-488.

[18] I.N. Katis, P.J.W. He, R.W. Eason, C.L. Sones, Improved sensitivity and limit-of-detection of lateral flow devices using spatial constrictions of the flow-path, Biosensors \& Bioelectronics. 113 (2018) 95-100. https://doi.org/10.1016/j.bios.2018.05.001.

[19] R. Ramamoorthy, V. Nallasamy, R. Reddy, N. Esther, Y. Maruthappan, A review of C-reactive protein: A diagnostic indicator in periodontal medicine, J. Pharm. Bioallied Sci. 4 (2012) 422. https://doi.org/10.4103/0975-7406.100318.

[20] Y. Luan, Y. Yao, The clinical significance and potential role of C-reactive protein in chronic inflammatory and neurodegenerative diseases, Frontiers in Immunology. 9 (2018) 1302.

[21] M.M. Ragy, N.N. Kamal, Linking senile dementia to type 2 diabetes: role of oxidative stress markers, C-reactive protein and tumor necrosis factor- $\alpha$, Neurological Research. 39 (2017) 587-595.

[22] M. Algarra, D. Gomes, J.C.G.E. da Silva, Current analytical strategies for C-reactive protein quantification in blood, Clinica Chimica Acta. 415 (2013) 1-9.

[23] C.M. McGeough, S. O'Driscoll, Camera phone-based quantitative analysis of C-reactive protein ELISA, IEEE Transactions on Biomedical Circuits and Systems. 7 (2013) 655-659.

[24] M. Dong, J. Wu, Z. Ma, H. Peretz-Soroka, M. Zhang, P. Komenda, N. Tangri, Y. Liu, C. Rigatto, F. Lin, Rapid and low-cost CRP measurement by integrating a paper-based microfluidic immunoassay with smartphone (CRP-Chip), Sensors. 17 (2017) 684. 
[25] P.M. Ridker, Clinical application of C-reactive protein for cardiovascular disease detection and prevention, Circulation. 107 (2003) 363-369.

[26] M.V. Humbert, L.E. Costa, I. Katis, F. Fonseca Ramos, A. Sanchéz Machado, C. Sones, E.A. Ferraz Coelho, M. Christodoulides, A rapid diagnostic test for human Visceral Leishmaniasis using novel Leishmania antigens in a Laser Direct-Write Lateral Flow Device, Emerging Microbes \& Infections. 8 (2019) 1178-1185.

[27] P.J.W. He, I.N. Katis, R.W. Eason, C.L. Sones, Rapid Multiplexed Detection on Lateral-Flow Devices Using a Laser Direct-Write Technique, Biosensors-Basel. 8 (2018). https://doi.org/10.3390/bios8040097.

[28] P.J.W. He, I.N. Katis, A.J.U. Kumar, C.A. Bryant, C.W. Keevil, B.K. Somani, N. Mahobia, R.W. Eason, C.L. Sones, Laser-patterned paper-based sensors for rapid point-of-care detection and antibiotic-resistance testing of bacterial infections, Biosensors and Bioelectronics. 152 (2020) 112008.

[29] Y.K. Oh, H.-A. Joung, S. Kim, M.-G. Kim, Vertical flow immunoassay (VFA) biosensor for a rapid one-step immunoassay, Lab on a Chip. 13 (2013) 768-772.

[30] J. Park, J.-K. Park, Pressed region integrated 3D paper-based microfluidic device that enables vertical flow multistep assays for the detection of $C$-reactive protein based on programmed reagent loading, Sensors and Actuators B: Chemical. 246 (2017) 1049-1055.

[31] E.G. Rey, D. O'Dell, S. Mehta, D. Erickson, Mitigating the hook effect in lateral flow sandwich immunoassays using real-time reaction kinetics, Analytical Chemistry. 89 (2017) 5095-5100.

[32] P.P. Galanis, P.J.W. He, I.N. Katis, M.R. Thomas, Y.L. Xianyu, M.M. Stevens, R.W. Eason, C.L. Sones, Laser direct-writing to enable filtration in paper-based devices, in: B.L. Gray, H. Becker (Eds.), Microfluidics, Biomems, and Medical Microsystems Xvii, Spie-Int Soc Optical Engineering, Bellingham, 2019. https://doi.org/10.1117/12.2508753.

[33] S.B. Bagadane, P.B. Jadhav, Development and validation of RP-HPLC method for simultaneous estimation of metformin hydrochloride and glipizide in bulk and pharmaceutical dosage form, Journal of Drug Delivery and Therapeutics. 9 (2019) 146-155. 